\title{
How I Treat Advanced Head Neck Cancer
}

\author{
Somnath Roy ${ }^{1}$ Joydeep Ghosh ${ }^{1}$ Sandip Ganguly ${ }^{1}$ \\ ${ }^{1}$ Department of Medical Oncology, Tata Medical Center, Newtown, \\ Kolkata, West Bengal, India \\ Ind J Med Paediatr Oncol
}

\section{Introduction}

In India, head and neck cancer including all sites is the most common cancer in terms of incidence and mortality among both sexes combined as per GLOBOCAN 2018, with lip and oral cavity being the most common site. ${ }^{1}$ Squamous cell cancer of head neck (HNSCC) constitutes the majority of all head and neck malignancies. ${ }^{2}$ Most of the patients present in the advanced stage. ${ }^{3}$ Even localized HNSCC are notorious for high rates of recurrence in spite of curative modalities of treatment. ${ }^{4}$ Treatment for advanced recurrent HNSCC is palliative systemic therapy provided that patients have a good Eastern Cooperative Oncology Group (ECOG) performance score (PS) with normal organ function.

Platinum-based systemic therapy is the standard of treatment in advanced metastatic HNSCC over a long period of time. Over time, antiepidermal growth factor receptor (EGFR) monoclonal antibody like cetuximab showed additional survival benefit over platinum-based therapy. ${ }^{5}$ Recently, immune-checkpoint inhibitors (IOs) have been found to benefit both in first- and second-line settings as a single agent or in combination with platinum-based therapy. ${ }^{6}$ Last but not the least, metronomic therapy has shown a survival benefit in recurrent metastatic settings. ${ }^{7}$ In addition to systemic therapy, supportive care in controlling the symptoms is absolutely essential in the management of advanced HNSCC. $^{4}$

India is a low middle-income country with a per capita income of Rs 11,254/- only. Majority of the people are not covered by health insurance. ${ }^{8}$ The cost of modern therapies like cetuximab and IOs are beyond the reach of most people from India. Hence, the treatment of any cancer including metastatic HNSCC has to be tailored based on the financial affordability of the patients and it should be used as an important factor to decide the systemic therapy.

DOI https://doi.org/ 10.1055/s-0042-1742320. ISSN 0971-5851.
Address for correspondence Sandip Ganguly, MD, DM (Medical Oncology), Consultant, Tata Medical Center, Department of Medical Oncology, 14 Mar EW Arterial Road, Newtown, Kolkata, West Bengal, India Pin 7000160 (e-mail: dr.babumashai@gmail.com).

\section{Case 1}

A 50-year-old male with a history of tobacco consumption presented with an ulcerated lesion over the lateral border of the tongue for the last 4 months. On clinical examination, there was an ulcerated lesion measuring $4 \times 4 \mathrm{~cm}$ with matted cervical lymphadenopathy. Biopsy of the lesion revealed a squamous cell carcinoma. Staging workup revealed multiple lung metastases. His ECOG PS was 1 and his organ functions were within normal limits. The patient could not afford the targeted therapy and immune checkpoint inhibition as his monthly salary was Rs $15,000 /-$ only. How to treat the patient?

The above patient was a case of de novo metastatic HNSCC. As the patient could not afford targeted therapies including immune checkpoint inhibitors, the patient could be treated with systemic therapy only. Historically, methotrexate alone was the standard systemic in recurrent metastatic HNSCC with a response rate of around 30\%. ${ }^{7}$ Phase II trials have shown that cisplatin alone had a similar response rate to single-agent methotrexate with increased toxicity. ${ }^{9}$ Carboplatin is a platinum analog with a better toxicity profile in contrast to cisplatin. Carboplatin has also shown a response rate of around $24 \%$ in the metastatic HNSCC with greater ease in administering the drug. ${ }^{10}$

Based on the encouraging response rates, a trial was made to use combination therapy in this setting. Phase III Southwest Oncology Group study was planned to see the effect of combination chemotherapy over single-agent methotrexate with the primary objective being to see response rate of doublet therapy over a single agent. It was a three-armed study. The study arms were a combination of cisplatin and 5 fluorouracil (5FU) and carboplatin and 5 FU with singleagent methotrexate being the control arm. The overall response rates were 32,21 , and $10 \%$, respectively, with the

(c) 2022. Indian Society of Medical and Paediatric Oncology. All rights reserved.

This is an open access article published by Thieme under the terms of the Creative Commons Attribution-NonDerivative-NonCommercial-License, permitting copying and reproduction so long as the original work is given appropriate credit. Contents may not be used for commercial purposes, or adapted, remixed, transformed or built upon. (https://creativecommons.org/ licenses/by-nc-nd/4.0/)

Thieme Medical and Scientific Publishers Pvt. Ltd., A-12, 2nd Floor, Sector 2, Noida-201301 UP, India 
difference in response rate between cisplatin and 5FU being statistically significant than single-agent methotrexate. ${ }^{11}$ Overall survival (OS) among the three arms were similar.

There was increased grade 3 and higher hematological toxicity along with renal toxicity in the cisplatin and 5FU arm. Based on this study, cisplatin and 5FU became the standard of therapy in metastatic HNSCC.

There was increased toxicity in the cisplatin and 5FU arm and it was attributed to the usage of 5FU. An attempt was made to replace 5FU with paclitaxel. Phase III trial (E1395) was planned to compare cisplatin and 5FU with a combination of cisplatin and paclitaxel. ${ }^{12}$ The response rate and OS in both the arms were comparable with less gastrointestinal and hematological toxicity in the paclitaxel arm.

Docetaxel was also used in combination with cisplatin in many phase II studies and they have shown response rates varying between 30 and $50 \%{ }^{13,14}$ No phase III trial has been conducted with this combination.

Pemetrexed-an antifolate analog-has been also tried in combination with cisplatin in advanced HNSCC. Phase III study was done to compare the efficacy of cisplatin pemetrexed over cisplatin alone. There was no added survival benefit with the doublet regime over single agent. But in post planned subset analysis, it was found that there was survival benefit in patients with oropharyngeal primary and with ECOG PS 0-1. ${ }^{15}$

In summary, combination chemotherapy has shown only improved response rate over single-agent therapy at an added cost of increased toxicity. None of the studies have shown OS benefit with combination therapy. ${ }^{10,16}$

\section{Case 2}

A 45-year-old female with a history of tobacco consumption presented with an ulcerated lesion over the buccal mucosa for the last 4 months. On clinical examination, there was an ulcerated lesion measuring $4 \times 4 \mathrm{~cm}$ and with matted cervical lymphadenopathy. Biopsy of the lesion showed squamous cell carcinoma. Staging workup revealed multiple lung metastases. Her ECOG PS is 1 and her organ functions are within normal limits. Patient could afford targeted therapy. How to treat the patient?

Treatment benefit with systemic chemotherapy alone is modest in metastatic HNSCC. Targeted therapies including monoclonal antibodies and very recently immunotherapies have shown improved survival benefit when compared with systemic therapy alone.

Tumor cells from HNSCC do express high levels of EGFR and it is associated with poor prognosis. ${ }^{17,18}$ Cetuximab is a chimeric monoclonal antibody that binds to EGFR receptor and causes cell death through antibody dependent cytotoxicity. It has been found to have a synergistic effect in combination with chemotherapeutic agents. ${ }^{19,20}$

In the landmark phase III EXTREME trial, cetuximab was used in combination with cisplatin 5FU and it was compared with the chemotherapy arm. ${ }^{5}$ It was found that there is an absolute OS advantage of 2.8 months (hazard ratio [HR]: 0.8; $95 \%$ confidence interval $[\mathrm{CI}]: 0.64-0.99 ; p=0.04)$ in the cetuximab arm. There was a higher incidence of hypomagnesemia, skin rash, infusion reaction, and sepsis related to cetuximab.

In view of concerns regarding toxicity with cisplatin $5 F U$, cetuximab has been used with taxane and cisplatin. In the TPExtreme trial, cetuximab was used with either cisplatin 5FU or cisplatin docetaxel. ${ }^{21}$ There was a similar OS in both the arms with less toxicity and reduced rates of discontinuation in the taxane combination group, thus making another alternative regime for metastatic HNSCC.

Panitumumab is a fully humanized monoclonal antibody against EGFR receptor. It has a similar mechanism of activity with cetuximab and has shown comparable results to cetuximab in metastatic colon cancer. ${ }^{22}$ It was expected that panitumumab will also show positive results in patients with metastatic HNSCC. But a phase III SPECTRUM trial that compared cisplatin 5FU with or without panitumumab failed to show an OS advantage in the arm containing panitumumab. ${ }^{23}$ Additionally, the panitumumab-based regime had a higher incidence of toxicity also. Only in unplanned subgroup analysis, the benefit of panitumumab was there in $\mathrm{p} 16$ negative and oral cavity tumors.

\section{Case 3}

A 56-year-old female with a history of tobacco consumption presented with an ulcerated lesion over the buccal mucosa for the last 4 months. On clinical examination, there was an ulcerated lesion measuring $4 \times 4 \mathrm{~cm}$ and with matted cervical lymphadenopathy. Biopsy of the lesion revealed squamous cell carcinoma with a combined positive score (CPS) score of $18 \%$. Staging workup revealed multiple lung metastases. Her ECOG PS was 1 and her organ functions were within normal limits. How to treat the patient?

IOs have been tried in metastatic HNSCC. For usage of IO in renal cancers and melanoma, no biomarker is required; while for usage in thoracic malignancies, program death ligand (PDL1) staining score is used as an indicator to use IO either as a single agent or in combination with chemotherapy. ${ }^{24}$ PDL1 staining is done on the tumor cells to calculate tumor proportion score (TPS) and various platforms have been used for the same. In HNSCC, instead of TPS scoring a CPS is calculated. In CPS scoring system, intensity is measured not only on the tumor cells but also on the lymphocytes and macrophages. CPS has been found to be a better predictor for response to IO. ${ }^{25}$

Pembrolizumab, a PD 1 inhibitor, has been approved to be used in the first line of metastatic HNSCC. It can be used either as a single agent or in combination with platinumbased therapy based on the phase III KEYNOTE 048 study. ${ }^{26}$ In this trial, de novo recurrent metastatic HNSCC patients irrespective of PDL1 score were randomized to three arms. They were pembrolizumab monotherapy, pembrolizumab with platinum and 5FU, and cetuximab with platinum and 5FU and the primary endpoint of the trial being OS. It was found that there was an OS advantage with either pembrolizumab alone or pembrolizumab with chemotherapy over 
the cetuximab chemotherapy combination in patients with CPS $>1$. However, OS benefit with pembrolizumab and chemotherapy over cetuximab-based combined therapy was persistent in the whole population irrespective of PDL1 status, but with pembrolizumab monotherapy, the OS was non inferior only to cetuximab arm. The median duration of response was prolonged in both the IO arms. Contrary to the OS advantage, the progression-free survival (PFS) advantage was not there in either the pembrolizumab alone or the combined arm over the chemotherapy. The added OS advantage in spite of inferior PFS was postulated to the alteration of the tumor microenvironment with better sensitivity to subsequent therapy. But this postulation is controversial at present.

The distinction to choose IO alone over combination of IO with chemotherapy in patients with CPS $>1$ is not very clear and it is arbitrarily based on the disease burden and symptom complex with pembrolizumab monotherapy is recommended for lesser disease burden with not much symptom complex.

\section{Case 4}

A 69-year-old male with a history of tobacco consumption presented with an ulcerated lesion over the gingival sulcus for last 4 months. On clinical examination, there was an ulceroproliferative lesion measuring $5 \times 5 \mathrm{~cm}$ and with matted cervical lymphadenopathy. Biopsy of the lesion was squamous cell carcinoma. Staging workup revealed multiple lung metastases. His ECOG PS is 2 and he was a coolie with a monthly income of around Rs 1,000 and he could not afford a daycare-based therapy. How to treat the patient?

Treatment of metastatic HNSCC is diverse and it ranges from systemic chemotherapy to monoclonal antibody and recently IOs. The last two options are very expensive in the setting of low middle-income countries like India, leading to less penetration to the patient population. The standard systemic chemotherapy has only shown a modest benefit with associated toxicity. Recently, metronomic therapy has been found to be beneficial in metastatic HNSCC.

Principle of metronomic therapy is repeated administration of selected chemotherapeutic agents at a low dose, which in turn inhibits angiogenesis and also has additional immunomodulatory effects leading to tumor control. ${ }^{27}$ Metronomic therapy has found to be beneficial in metastatic HNSCC also. ${ }^{28}$ Various agents have been used, most commonly being methotrexate and celecoxib and most were in phase I to II studies. Oral capecitabine has also been used in some cases. Only recently, a phase III study was conducted where metronomic therapy was compared with single-agent cisplatin with the primary objective being OS benefit of metronomic therapy over chemotherapy. ${ }^{7}$ Here, patients were randomized to receive either intravenous cisplatin or combination of methotrexate and celecoxib. It was found that median OS in the metronomic arm was superior than the chemotherapy arm (HR: $0.773 ; 95 \% \mathrm{CI}: 0.65-0.97 ; p=0.026$ ) with a better toxicity profile in the metronomic arm. The choice of chemotherapy in the control arm is justifiable as no studies have shown a better OS with doublet chemotherapy over single chemotherapeutic agent.

\section{Case 5}

A 60-year-old male presented with history of tobacco consumption presented with an ulcerated lesion over the gingival sulcus for the last 4 months. On clinical examination, there was an ulceroproliferative lesion measuring $5 \times 5 \mathrm{~cm}$ with matted cervical lymphadenopathy. Biopsy of the lesion revealed squamous cell carcinoma. Staging workup revealed multiple lung metastases. He was treated with a combination of cisplatin and $5 \mathrm{FU}$ for a total of 6 cycles. Unfortunately, he developed a progressive disease within 6 months. What are the options to treat the patient?

The choice of second line therapy depends upon the PS of the patient, prior systemic therapy, platinum free interval, and most importantly the financial status. Platinum-free interval (PFI) is defined as the time interval between the last platinum-based therapy to the development of disease progression. The time interval ranges from 3 to 6 months as mentioned in different clinical trials. ${ }^{29,30}$ Those who have a PFI of more than 3 months can be rechallenged with platinum-based therapy. The treatment options are discussed as follows:

\section{A. Systemic chemotherapy}

In patients with PFI of more than 6 months, patients can be rechallenged with platinum-based therapy either alone or in combination with doublet-based chemotherapy. ${ }^{9,31}$ Another option is to give low-dose metronomic chemotherapy with methotrexate and celecoxib as it has shown superior survival over cisplatin. ${ }^{7}$

Patients who had shorter PFI are deemed to be defined as platinum resistant and carry a poor prognosis. Agents that have been tried are docetaxel and methotrexate. Response rates and OS are very modest and there was no survival advantage over one another. ${ }^{32}$

B. Cetuximab

In patients with no prior exposure to cetuximab and with PFI of more than 6 months, they can be treated with cetuximab along with platinum doublet therapy. ${ }^{5}$

Cetuximab has been used either as a single agent or in combination with platinum in patients with shorter PFT in small phase II studies. As a single agent, response rate is around $13 \%$ with a median OS of 178 days. ${ }^{33}$ Cetuximab has been tried in combination with platinum in a platinum resistant population. The response rates were almost similar to cetuximab monotherapy. ${ }^{34}$

\section{Immunotherapy}

Before the advent of immunotherapy as a first-line therapy in metastatic HNSCC, they were tried in recurrent second line settings and the results were very encouraging.

Both pembrolizumab and nivolumab have received U.S. Food and Drug Administration approval for usage in the second line setting. 
Head Neck Cancer, Chemotherapy, Immunotherapy, Cetuximab Roy et al.

Pembrolizumab anti-PD1 inhibitor was compared with nonplatinum agents in platinum resistant cases in KEYNOTE-040 trial. ${ }^{30}$ There was an OS advantage in the whole population with pembrolizumab over the controlled arm (HR: $0.8,0.65-0.98 ; p=0.016$ ), but mainly in patients with PDL1 $\geq$ to $50 \%$ (HR: $0.53 ; 0.35-0.81$ ), the response rates with pembrolizumab were modestly superior than the control arm with lesser incidence of adverse events in the pembrolizumab arm compared with the control arm (13 vs. $36 \%$, respectively).

Nivolumab was also used in a similar way in platinum resistant cases in phase III checkmate 141 trial. $^{29}$ The primary endpoint was again OS. The trial met its primary in point of improvement of OS from 5.1 to 7.5 months with the use of nivolumab (HR for death, $0.70 ; 97.73 \% \mathrm{CI}: 0.51-$ $0.96 ; p=0.01$ ), and the 1 -year survival was 36 vs. $16.6 \%$. This trial again established the role of immunotherapy by improving OS in the second line.

Durvalumab, an anti PDL1 inhibitor, has also been tried in recurrent metastatic HNSCC. In a single-arm phase II study, there was a response rate of around $16 \%$ with OS of 7.1 months. ${ }^{35}$ Durvalumab was compared alone or in combination with tremelimumab with standard systemic therapy in phase III study but it failed to show any benefit over standard systemic therapy. ${ }^{36}$

D. EGFR Tyrosine Kinase Inhibitor

EGFR tyrosine kinase inhibitors like gefitinib and afatinib have been used in platinum resistant settings. Gefitinib

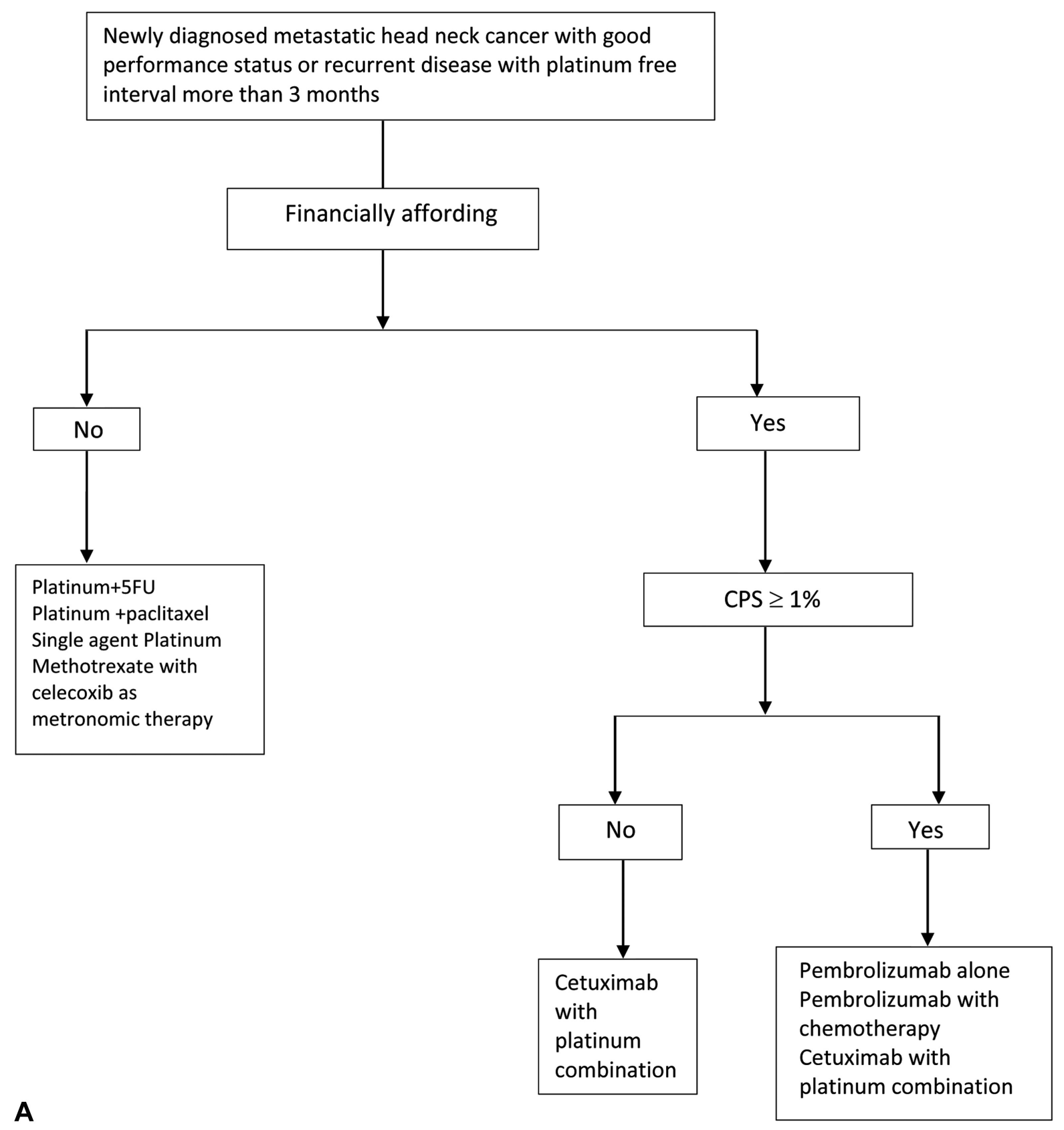

Fig. 1 (A, B) Algorithm regarding first-line treatment in newly diagnosed/-platinum-sensitive recurrent metastatic head neck cancer. 


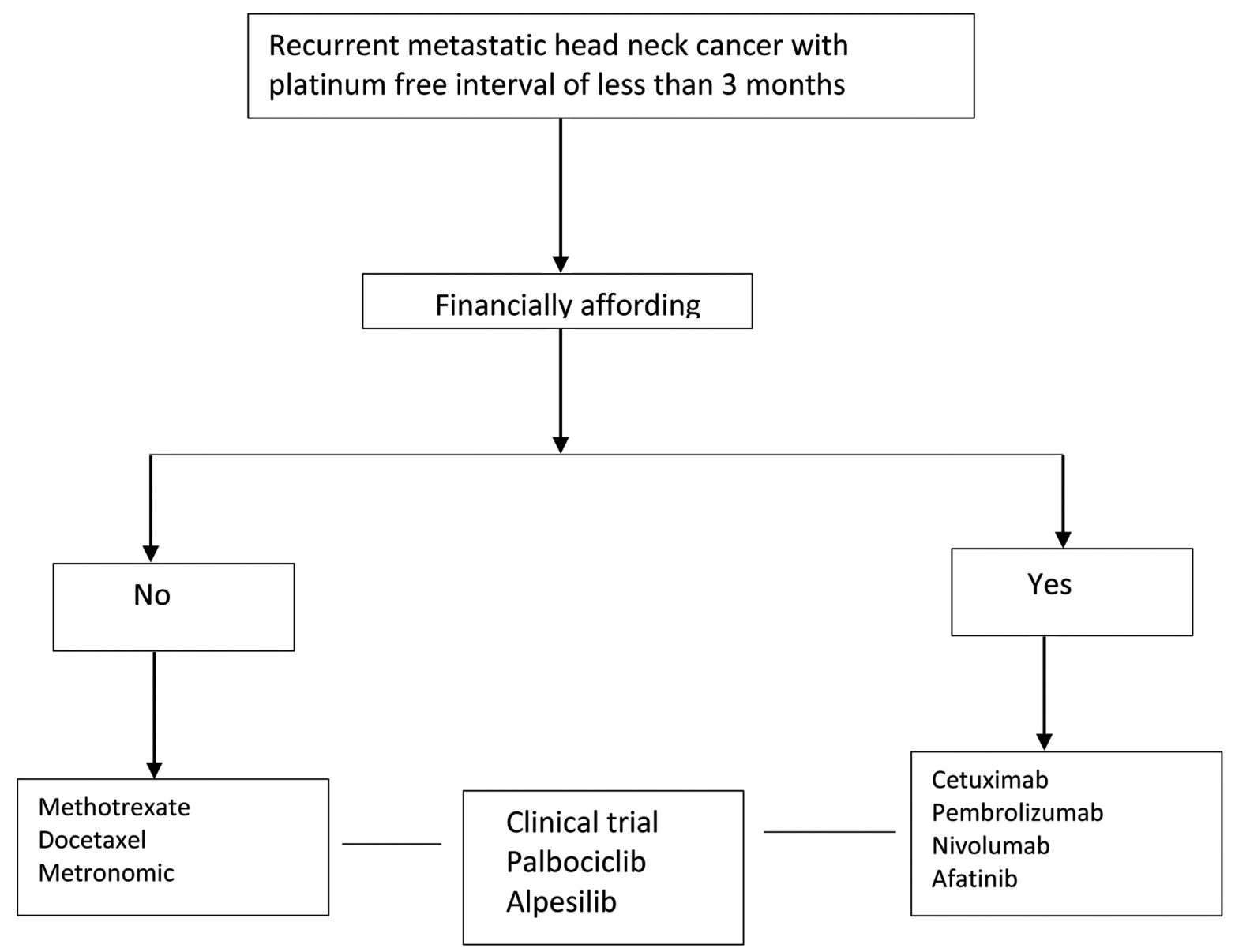

Fig. 1 (Continued)

did not improve either the response rate or survival compared with single-agent methotrexate. ${ }^{37}$ Afatinib was compared with intravenous methotrexate (40 $\mathrm{mg} / \mathrm{m}^{2} /$ week) in a phase III randomized trial involving Asian patients who had the disease progression following first-line platinum-based chemotherapy.

Out of the 340 randomized patients, afatinib significantly decreased the risk of progression or death by $37 \%$ versus methotrexate (HR: $0.63 ; 95 \%$ CI: $0.48-0.82 ; p=0.0005$; median 2.9 vs. 2.6 months). The response rate was $28 \%$ with afatinib versus $13 \%$ with methotrexate. ${ }^{38}$ Another EGFR tyrosine kinase inhibitor erlotinib has been tried in combination with celecoxib and methotrexate as a metronomic therapy in platinum resistant oral cancers. The 3 month PFS was $71.1 \%$ and the 6 month OS was $61.2 \%$ with a response rate of $43 \%{ }^{39}$

E. Newer therapies

The field of recurrent metastatic head and the cancer is evolving fast with newer developments largely based on molecular pathways. Similar pathway is the CDK4/6 pathway. The CDK 4/6 inhibitor palbociclib has been investigated in phase I and II trials. In a phase II trial in combination with cetuximab, the objective response rate was $39 \%$ in the platinum resistant group. ${ }^{40,41}$ Buparlisib $100 \mathrm{mg}$ once daily, a phosphoinositide 3 kinase (Pi3K) inhibitor, was combined with paclitaxel versus paclitaxel alone in the phase II randomized BERIL 1 trial. There was an absolute 1.1-month PFS benefit in the combination arm (HR: $-0.65 ; 95 \%$ CI: $0.45-0.95 ; p=0.01) .{ }^{42}$ Alpelisib is also being studied actively in such patients. ${ }^{43}$

Armamentarium of systemic therapy in metastatic HNSCC has been steadily increased over with the addition of monoclonal antibodies including IO. The response rate and survival advantage is still modest despite being statistically significant. Unfortunately, many of the therapies are still out of the reach for the common people depriving them to get these newer therapies. The therapeutic modality should be tailored and we proposed algorithm as shown in - Fig. $\mathbf{1 A}$ and $\mathbf{B}$, respectively.

\section{Case 6}

A 25-year-old female from Manipur presented with painless lump over both side of her neck with nasal obstruction since last 3 months. It was neither associated with any fever, cough, night sweats nor any other systemic symptoms. On clinical examination, there was $3 \times 4 \mathrm{~cm}$ lymph node at right level III and multiple neck nodes max. $3.5 \mathrm{~cm}$ in greatest dimension at left level II, III, and IV region. On nasal endoscope, there was mass lesion arising from fossa of Rosenmollar extending right posterior nasal cavity. Biopsy from the mass lesion revealed nonkeratinizing squamous cell carcinoma, 
undifferentiated subtype; the tumor cells were positive for p63 and cytokeratin.

Tumor cells are POSITIVE for Epstein-Barr virus encoded RNA (EBER-ISH) consistent with nasopharyngeal primary. Magnetic resonance imaging neck showed $6 \times 4.5 \mathrm{~cm}$ mass arising from fossa of Rosenmollar with obliteration of left parapharyngeal fat and extending into anterior nasal cavity; without any intracranial extension, enlarged bilateral level II, III, IV, and V cervical nodes maximum dimension $5.2 \mathrm{~cm}$ with small right retropharyngeal node. Positron emission tomography computed tomography (PET CT) did not reveal any other metabolically active disease noted elsewhere. After 8 months of completion of her treatment, PET CT showed multiple lung metastasis; she was otherwise fit and symptomatic for her lung metastasis. How to treat the patient?

Nasopharyngeal cancer (NPC) is a malignancy with highly curative potential; skewed geographical distribution and mostly present among younger population with locally advanced stage. Infection with EBV is an important etiological factor particularly in nonkeratinizing and undifferentiated subtype. Workup for NPC should include EBV testing from both tumor and the blood. The method of detection of EBV in tumor includes In situ hybridization (ISH) for EBV encoded RNA (EBER) and IHC staining for LMP1. ${ }^{44,45}$ Real-time polymerase chain reaction is used to evaluate EBV DNA load in plasma or serum and used as a marker for residual diseases monitoring. A meta-analysis showed that pretreatment plasma EBV DNA levels were independent prognostic factors for mortality and distant metastasis. ${ }^{46}$

Patients with early stage T1N0 M0 NPC should be treated with definitive radiation alone with local control rate around 90\%. ${ }^{47}$ For loco regionally advanced stage, the Intergroup trial 099 showed highly significant survival advantage favoring combined modality with cisplatin-based chemotherapy and radiation. ${ }^{48}$ Asia-specific phase III randomized controlled trails (RCTs) confirmed that 5 years OS was around $70 \%$ for the chemoradiation compared with RT alone. ${ }^{49}$ Subsequently, an individual patient-based meta-analysis of eight RCTs showed an absolute benefit in OS and EFS at 5 years with highest benefit resulting from concomitant chemoradiation rather than neoadjuvant or adjuvant chemotherapy. ${ }^{50}$ A network meta-analysis (including 20 trials and 5,144 patient) showed that addition of adjuvant chemotherapy to chemoradiation was associated with better PFS compared with chemoradiation alone. ${ }^{51}$

Meta-analysis established the role of induction chemotherapy followed by chemoradiation is another standard of care in node-positive diseases. ${ }^{52}$ Gemcitabine and cisplatin are preferred induction regimen, whereas for EBV-associated diseases modified TPF (docetaxel $60 \mathrm{mg} / \mathrm{m}^{2}$, cisplatin $60 \mathrm{mg} / \mathrm{m}^{2}, 5 \mathrm{FU}$ $600 \mathrm{mg} / \mathrm{m}^{2} \mathrm{CI}$ for 5 days) is preferred regimen. ${ }^{53,54}$

For metastatic NPC, there is limited options for systemic therapy and enrolment in a clinical trials is preferred. Gemcitabine plus cisplatin is the preferred first-line chemotherapy regimen demonstrated survival advantage compared with Cisplatin plus $5 \mathrm{FU} .^{55}$ The role of cetuximab in combination with platinum salts tested in a phase II trial with acceptable safety profile in heavily pretreated patients. ${ }^{56}$ The role immune checkpoint inhibitors like pembrolizumab (KEYNOTE028) and nivolumab ( NCI 9742) were tested in phase I/II trials, but the results were not so encouraging. ${ }^{57,58}$

Conflict of Interest

None.

\section{References}

1 356-india-fact-sheets.pdf [Internet]. [cited 2020 Sep 6]. Accessed December 7, 2021: https://gco.iarc.fr/today/data/factsheets/populations/356-india-fact-sheets.pdf

2 Shah SB, Sharma S, D'Cruz AK. Head and neck oncology: the Indian scenario. South Asian J Cancer 2016;5(03):104-105

3 Mohanti BK, Nachiappan P, Pandey RM, Sharma A, Bahadur S, Thakar A. Analysis of 2167 head and neck cancer patients management, treatment compliance and outcomes from a regional cancer centre, Delhi, India. J Laryngol Otol 2007;121(01): 49-56

4 Parikh P, Patil V, Agarwal JP, et al; Indian Cooperative Oncology Network. Guidelines for treatment of recurrent or metastatic head and neck cancer. Indian J Cancer 2014;51(02):89-94

5 Vermorken JB, Mesia R, Rivera F, et al. Platinum-based chemotherapy plus cetuximab in head and neck cancer. N Engl J Med 2008;359(11):1116-1127

6 Ferris RL. Immunology and immunotherapy of head and neck cancer. J Clin Oncol 2015;33(29):3293-3304

7 Patil V, Noronha V, Dhumal SB, et al. Low-cost oral metronomic chemotherapy versus intravenous cisplatin in patients with recurrent, metastatic, inoperable head and neck carcinoma: an open-label, parallel-group, non-inferiority, randomised, phase 3 trial. Lancet Glob Health 2020;8(09):e1213-e1222

8 Ministry of Statistics and Program Implementation | Government Of India [Internet]. [cited 2020 Oct 13]. Accessed December 7, 2021: http://www.mospi.gov.in/

9 Grose WE, Lehane DE, Dixon DO, Fletcher WS, Stuckey WJ. Comparison of methotrexate and cisplatin for patients with advanced squamous cell carcinoma of the head and neck region: a Southwest Oncology Group Study. Cancer Treat Rep 1985;69(06):577-581

10 Al-Sarraf M, Metch B, Kish J, et al. Platinum analogs in recurrent and advanced head and neck cancer: a Southwest Oncology Group and Wayne State University Study. Cancer Treat Rep 1987;71(78):723-726

11 Forastiere AA, Metch B, Schuller DE, et al. Randomized comparison of cisplatin plus fluorouracil and carboplatin plus fluorouracil versus methotrexate in advanced squamous-cell carcinoma of the head and neck: a Southwest Oncology Group study. J Clin Oncol 1992;10(08):1245-1251

12 Gibson MK, Li Y, Murphy B, et al; Eastern Cooperative Oncology Group. Randomized phase III evaluation of cisplatin plus fluorouracil versus cisplatin plus paclitaxel in advanced head and neck cancer (E1395): an intergroup trial of the Eastern Cooperative Oncology Group. J Clin Oncol 2005;23(15):3562-3567

13 Schöffski P, Catimel G, Planting AST, et al. Docetaxel and cisplatin: an active regimen in patients with locally advanced, recurrent or metastatic squamous cell carcinoma of the head and neck. Results of a phase II study of the EORTC early clinical studies group. Ann Oncol 1999;10(01):119-122

14 Baur M, Kienzer H-R, Schweiger J, et al. Docetaxel/cisplatin as first-line chemotherapy in patients with head and neck carcinoma: a phase II trial. Cancer 2002;94(11):2953-2958

15 Urba S, van Herpen CML, Sahoo TP, et al. Pemetrexed in combination with cisplatin versus cisplatin monotherapy in patients with recurrent or metastatic head and neck cancer: final results of a randomized, double-blind, placebo-controlled, phase 3 study. Cancer 2012;118(19):4694-4705 
16 Williams SD, Velez-Garcia E, Essessee I, Ratkin G, Birch R, Einhorn LH. Chemotherapy for head and neck cancer. Comparison of cisplatin + vinblastine + bleomycin versus methotrexate. Cancer 1986;57(01):18-23

17 Levels of TGF- $\alpha$ and EGFR Protein in Head and Neck Squamous Cell Carcinoma and Patient Survival. | JNCI: Journal of the National Cancer Institute | Oxford Academic [Internet]. [cited 2020 Sep 13]. Accessed December 7, 2021: https://academic.oup.com/jnci/article/90/11/824/916287

18 Chung CH, Ely K, McGavran L, et al. Increased epidermal growth factor receptor gene copy number is associated with poor prognosis in head and neck squamous cell carcinomas. J Clin Oncol 2006;24(25):4170-4176

19 Fan Z, Baselga J, Masui H, Mendelsohn J. Antitumor effect of antiepidermal growth factor receptor monoclonal antibodies plus cisdiamminedichloroplatinum on well established A431 cell xenografts. Cancer Res 1993;53(19):4637-4642

20 Kang X, Patel D, Ng S, Melchior M, Ludwig D, Hicklin J. High affinity Fc receptor binding and potent induction of antibody-dependent cellular cytotoxicity (ADCC) in vitro by anti-epidermal growth factor receptor antibody cetuximab. J Clin Oncol 2007;25(18):3041-3041

21 Guigay J, Fayette J, Mesia R, et al. TPExtreme randomized trial: TPEx versus Extreme regimen in 1st line recurrent/metastatic head and neck squamous cell carcinoma (R/M HNSCC). J Clin Oncol 2019;37(15):6002-6002

22 Weber J, McCormack PL. Panitumumab: in metastatic colorectal cancer with wild-type KRAS. BioDrugs 2008;22(06):403-411

23 Vermorken JB, Stöhlmacher-Williams J, Davidenko I, et al; SPECTRUM investigators. Cisplatin and fluorouracil with or without panitumumab in patients with recurrent or metastatic squamous-cell carcinoma of the head and neck (SPECTRUM): an open-label phase 3 randomised trial. Lancet Oncol 2013;14(08): 697-710

24 Ancevski Hunter K, Socinski MA, Villaruz LC. PD-L1 testing in guiding patient selection for PD-1/PD-L1 inhibitor therapy in lung cancer. Mol Diagn Ther 2018;22(01):1-10

25 Bauml JM, Aggarwal C, Cohen RB. Immunotherapy for head and neck cancer: where are we now and where are we going? Ann Transl Med 2019;7(3, Suppl 3)S75

26 Pembrolizumab alone or with chemotherapy versus cetuximab with chemotherapy for recurrent or metastatic squamous cell carcinoma of the head and neck (KEYNOTE- 048): a randomised, open-label, phase 3 study. - The Lancet [Internet] [cited 2020 Sep 20]. Accessed December 7, 2021: https://www.thelancet.com/journals/lancet/article/PIIS0140-6736(19)32591-7/fulltext

27 Dabkara D, Ganguly S, Biswas B, Ghosh J. Metronomic therapy in metastatic castrate-resistant prostate cancer: experience from a tertiary cancer care center. Indian J Cancer 2018;55(01):94-97

28 De Felice F, Musio D, Tombolini V. Head and neck cancer: metronomic chemotherapy. BMC Cancer 2015;15:677 https://www.ncbi. nlm.nih.gov/pmc/articles/PMC4603691/ Accessed December 7, 2021 [Internet]

29 Ferris RL, Licitra L, Fayette J, et al. Nivolumab in patients with recurrent or metastatic squamous cell carcinoma of the head and neck: efficacy and safety in CheckMate 141 by prior cetuximab use. Clin Cancer Res 2019;25(17):5221-5230

30 Cohen EEW, Soulières D, Le Tourneau C, et al; KEYNOTE-040 investigators. Pembrolizumab versus methotrexate, docetaxel, or cetuximab for recurrent or metastatic head-and-neck squamous cell carcinoma (KEYNOTE-040): a randomised, open-label, phase 3 study. Lancet 2019;393(10167):156-167

31 Pêtre A, Dalban C, Karabajakian A, et al. Carboplatin in combination with weekly paclitaxel as first-line therapy in patients with recurrent/metastatic head and neck squamous cell carcinoma unfit to EXTREME schedule. Oncotarget 2018;9(31):22038-22046

32 Rapidis A, Sarlis N, Lefebvre J-L, Kies M. Docetaxel in the treatment of squamous cell carcinoma of the head and neck. Ther Clin Risk Manag 2008;4(05):865-886
33 Vermorken JB, Trigo J, Hitt R, Koralewski P, Diaz-rubio E, Rolland F, Knecht R, Amellal N, Schueler A, Baselga J. Open-label, uncontrolled, multicenter phase II study to evaluate the efficacy and toxicity of cetuximab as a single agent in patients with recurrent and/or metastatic squamous cell carcinoma of the head and neck who failed to respond to platinum-based therapy. J Clin Oncol 2007 Jun 1;25(16):2171-2177

34 Baselga J, Trigo JM, Bourhis J, et al. Phase II multicenter study of the antiepidermal growth factor receptor monoclonal antibody cetuximab in combination with platinum-based chemotherapy in patients with platinum-refractory metastatic and/or recurrent squamous cell carcinoma of the head and neck. J Clin Oncol 2005; 23(24):5568-5577

35 Zandberg DP, Algazi AP, Jimeno A, et al. Durvalumab for recurrent or metastatic head and neck squamous cell carcinoma: results from a single-arm, phase II study in patients with $\geq 25 \%$ tumour cell PD-L1 expression who have progressed on platinum-based chemotherapy. Eur J Cancer 2019;107(107):142-152

36 Ferris RL, Haddad R, Even C, et al. Durvalumab with or without tremelimumab in patients with recurrent or metastatic head and neck squamous cell carcinoma: EAGLE, a randomized, open-label phase III study. Ann Oncol 2020;31(07):942-950

37 Stewart JSW, Cohen EEW, Licitra L, et al. Phase III study of gefitinib compared with intravenous methotrexate for recurrent squamous cell carcinoma of the head and neck [corrected]. [corrected]J Clin Oncol 2009;27(11):1864-1871

38 Guo Y, Ahn M-J, Chan A, et al. Afatinib versus methotrexate as second-line treatment in Asian patients with recurrent or metastatic squamous cell carcinoma of the head and neck progressing on or after platinum-based therapy (LUX-Head \& Neck 3): an open-label, randomised phase III trial. Ann Oncol 2019;30 (11):1831-1839

39 Patil VM, Noronha V, Joshi A, et al. Phase I/II Study of Palliative Triple Metronomic Chemotherapy in Platinum-Refractory/EarlyFailure Oral Cancer. J Clin Oncol 2019;37(32):3032-3041

40 Michel L, Ley J, Wildes TM, et al. Phase I trial of palbociclib, a selective cyclin dependent kinase 4/6 inhibitor, in combination with cetuximab in patients with recurrent/metastatic head and neck squamous cell carcinoma. Oral Oncol 2016;58:41-48

41 Adkins D, Ley J, Neupane P, et al. Palbociclib and cetuximab in platinum-resistant and in cetuximab-resistant human papillomavirus-unrelated head and neck cancer: a multicentre, multigroup, phase 2 trial. Lancet Oncol 2019;20(09):1295-1305

42 Soulières D, Faivre S, Mesía R, et al. Buparlisib and paclitaxel in patients with platinum-pretreated recurrent or metastatic squamous cell carcinoma of the head and neck (BERIL-1): a randomised, double-blind, placebo-controlled phase 2 trial. Lancet Oncol 2017;18(03):323-335

43 Keam B, Kim S, Ahn Y-O, et al. In vitro anticancer activity of PI3K alpha selective inhibitor BYL719 in head and neck cancer. Anticancer Res 2015;35(01):175-182

44 Zeng Z, Fan S, Zhang X, et al. Epstein-Barr virus-encoded small RNA 1 (EBER-1) could predict good prognosis in nasopharyngeal carcinoma. Clin Transl Oncol 2016;18(02):206-211

45 Jeon YK, Lee BY, Kim JE, Lee SS, Kim CW. Molecular characterization of Epstein-Barr virus and oncoprotein expression in nasopharyngeal carcinoma in Korea. Head Neck 2004;26(07):573-583

46 Zhang W, Chen Y, Chen L, et al. The clinical utility of plasma Epstein-Barr virus DNA assays in nasopharyngeal carcinoma: the dawn of a new era?: a systematic review and meta-analysis of 7836 cases Medicine (Baltimore) 2015;94(20):e845

47 Lee AW, Ng WT, Chan LL, et al. Evolution of treatment for nasopharyngeal cancer-success and setback in the intensity-modulated radiotherapy era. Radiother Oncol 2014;110(03):377-384

48 Al-Sarraf M, LeBlanc M, Giri PG, et al. Chemoradiotherapy versus radiotherapy in patients with advanced nasopharyngeal cancer: phase III randomized Intergroup study 0099. J Clin Oncol 1998;16 (04):1310-1317 
49 Chan AT, Leung SF, Ngan RK, et al. Overall survival after concurrent cisplatin-radiotherapy compared with radiotherapy alone in locoregionally advanced nasopharyngeal carcinoma. J Natl Cancer Inst 2005;97(07):536-539

50 Baujat B, Audry H, Bourhis J, et al; MAC-NPC Collaborative Group. Chemotherapy in locally advanced nasopharyngeal carcinoma: an individual patient data meta-analysis of eight randomized trials and 1753 patients. Int J Radiat Oncol Biol Phys 2006;64 (01):47-56

51 Ribassin-Majed L, Marguet S, Lee AWM, et al. What is the best treatment of locally advanced nasopharyngeal carcinoma? An individual patient data network meta-analysis. J Clin Oncol 2017; 35(05):498-505

52 Wang M, Tian H, Li G, et al. Significant benefits of adding neoadjuvant chemotherapy before concurrent chemoradiotherapy for locoregionally advanced nasopharyngeal carcinoma: a metaanalysis of randomized controlled trials. Oncotarget 2016;7(30): 48375-48390

53 Zhang Y, Chen L, Hu GQ, et al. Gemcitabine and cisplatin induction chemotherapy in nasopharyngeal carcinoma. N Engl J Med 2019; 381(12):1124-1135
54 Sun Y, Li WF, Chen NY, et al. Induction chemotherapy plus concurrent chemoradiotherapy versus concurrent chemoradiotherapy alone in locoregionally advanced nasopharyngeal carcinoma: a phase 3, multicentre, randomised controlled trial. Lancet Oncol 2016;17(11):1509-1520

55 Zhang L, Huang Y, Hong S, et al. Gemcitabine plus cisplatin versus fluorouracil plus cisplatin in recurrent or metastatic nasopharyngeal carcinoma: a multicentre, randomised, open-label, phase 3 trial. Lancet 2016;388(10054):1883-1892

56 Chan AT, Hsu MM, Goh BC, et al. Multicenter, phase II study of cetuximab in combination with carboplatin in patients with recurrent or metastatic nasopharyngeal carcinoma. J Clin Oncol 2005;23(15):3568-3576

57 Hsu C, Lee SH, Ejadi S, et al. Safety and antitumor activity of pembrolizumab in patients with programmed death-ligand 1positive nasopharyngeal carcinoma: results of the KEYNOTE-028 study. J Clin Oncol 2017;35(36):4050-4056

58 Ma BBY, Lim WT, Goh BC, et al. Antitumor activity of nivolumab in recurrent and metastatic nasopharyngeal carcinoma: an International, Multicenter Study of the Mayo Clinic Phase 2 Consortium (NCI-9742). J Clin Oncol 2018;36(14):1412-1418 